\title{
Correction to: Manufacturing Genome: A Foundation for Symbiotic, Highly Iterative Product and Production Adaptations
}

Patrizia Gartner, Alexander Jacob, Haluk Akay, Johannes Löffler, Jack Gammack, Gisela Lanza, and Sang-Gook Kim

\author{
Correction to: \\ Chapter "Manufacturing Genome: A Foundation \\ for Symbiotic, Highly Iterative Product and Production \\ Adaptations" in: A.-L. Andersen et al. (Eds.): \\ Towards Sustainable Customization: Bridging Smart Products \\ and Manufacturing Systems, LNME, \\ https://doi.org/10.1007/978-3-030-90700-6_3
}

In the original version of the book, the following belated correction has been incorporated: In the chapter "Manufacturing Genome: A Foundation for Symbiotic, Highly Iterative Product and Production Adaptations", the affiliation of Patrizia Gartner has been changed from "Institute of Production Science, Karlsruhe Institute of Technology (KIT), Kaiserstr. 12, 76131 Karlsruhe, Germany" to "Department of Mechanical Engineering, Massachusetts Institute of Technology (MIT), 77 Massachusetts Avenue, Cambridge, MA 02139, USA". 